\title{
A Value-sensitive Toolkit: Bringing Values into the Design Process when Designing for the Elderly
}

\author{
Mert Oktay ${ }^{*}, \dagger$ and Hanna-Liisa Pender ${ }^{*}, \dagger$ \\ *Tallinn University, Narva rd 25, 10120 Tallinn, Estonia \\ †Trinidad Wiseman, Akadeemia rd. 21, 12618 Tallinn, Estonia \\ merdo@tlu.ee,pender@tlu.ee \\ www.tlu.ee, www.twn.ee
}

\begin{abstract}
The paper gives and overview of developing a design toolkit for designers that would encourage them to keep the human values on the forefront, despite all the other constraints that need to be faced when designing new technologies. The toolkit was validated and refined in a series of workshops with designers and design students. The outcome of the work is a toolkit prototype that includes tools like design fiction, bootlegging and value review. It is intended to be used to compliment a human centred design process after user research to scaffold ideation and tackle design challenges related to aging in place and smart habitats for elderly.
\end{abstract}

\section{Keywords}

Value based design · design fiction · elderly $\cdot$ design methods

How to cite this book chapter:

Oktay, M. and Pender, H-L. 2020. A Value-sensitive Toolkit: Bringing Values into the Design Process when Designing for the Elderly. In: Loizides, F., Winckler, M., Chatterjee, U., Abdelnour-Nocera, J. and Parmaxi, A. (eds.) Human Computer Interaction and Emerging Technologies: Adjunct Proceedings from the INTERACT 2019 Workshops. Pp. 23-30. Cardiff: Cardiff University Press. DOI: https://doi. org/10.18573/book3.c. License: CC-BY 4.0. 


\section{Introduction}

In the industry context, user research is an essential part of the whole design journey, however, after user research there is often relatively little attention on the ideation process. The solutions are in many cases technology driven with too many material constraints or the most doable ones in a short time period. There are numerous ideation tools, commonly used in industry context, that are focused on human values e.g empathy maps [1], value proposition maps [2]; however none of them aim for value-sensitive solutions. Even though these tools might be efficient to map user needs and desires; they do not guide designers to think about possible solutions. While the needs and values can be well-described, the tendency of design teams is still to choose the most obvious and quickly-doable solution. Thus, there is a room for an additional toolkit which leads designers to develop meaningful and value-sensitive design solutions.

The context of this study is a contemporary issue that worries the entire world, especially the Global North: the population aging. According to research of European Commission; a quarter of Europeans will be over 60 years of age by 2020 . This situation brings with it some concerns related to elderly care and independence of the elderly. Thereby, aging in place term [8] which provides autonomy for elderly, becomes more and more popular [9]. Elderly prefer to remain in the comfort of their familiar and safe homes instead of moving to a geriatric care center [10] which might create a negative emotional impact, both on the elderly and their families. Smart home technologies could be useful to support and to enhance aging in place for the elderly, although, these technologies might create privacy issues because they mostly rely on constant monitoring [11]. Privacy is only one of the many human values that designers should take into consideration while creating more meaningful solutions for the elderly. We are seeing more and more smart solutions that aim to support independent living for elderly whos physical and mental abilities are declining. These are for example smart habitats that involve constant medical monitoring of people's health. Technology is reaching for the most intimate spheres of human existence. That is why, it is crucial to consider the non-functional aspects of new technologies, when designing them. By empathizing with the user and designing from their perspective, designers can create solutions that people are more likely to adopt and appreciate. There are several studies $[12,13]$ focused on design values to use while designing for the elderly, however, they are not structured and formulated to be a guideline that would support designers, especially in industry context where designers need to often account with very limited time. Therefore a ideation toolkit for designers is proposed, that aims to endorse value based design for elderly. This paper gives an overview of developing a such toolkit and the lessons learned during the process. 


\section{Method}

Research Goal: The goal of this research was to develop a value-sensitive design toolkit that would help to design for the elderly. To achieve these goals, two main research questions were selected as starting points.

[RQ1] What are the main design principles and values when designing for elderly?

The first step of the research was identifying design values that are used when designing for elderly. A systematic literature review was selected as method to identify the main design principles and how the value-sensitive concerns are addressed today when designing technology for the aging people.

[RQ2] How can the design values be meaningfully conveyed into the design process with the help of a design toolkit?

To answer this research question, research through design approach was adopted as the overall methodology. For achieving the goal, designing a meaningful and useful value-sensitive design toolkit that can be used to emphasize values when designing for elderly, an iterative approach was used. The proposed design toolkit prototype was refined in workshops with the help of designers and design students. Four ideation workshops were conducted in order to evaluate and improve the design toolkit.

Since research through design also asks researchers to investigate the speculative future, probing on what the world could and should be [3], this methodology suits this study in which a critical approach was adopted by practicing design fiction.

\section{Developing the design toolkit}

\subsection{Literature review to map the values used in designing for elderly}

A literature review was conducted to gain an overview of state of the art in designing for elderly care related challenges. 80 research papers were examined from ACM Digital Library. The systematic literature review consists of independent aging for the elderly and our keywords were elderly, elderly care, aging in place, elderly independence, smart homes for elderly, e lderly monitoring. The oldest publication included in the corpus was from 2005, the newest from 2018. All the papers were reviewed using the quick and dirty method [14]. Papers that addressed value-sensitive concerns e.g privacy issues became prominent. Thereby, papers addressing the value-sensitive concerns in the context of the independent aging, and the ones that give example use cases of smart home technologies in the aging in place context received the highest interest rating. A qualitative content analysis was conducted on 32 papers that received the highest interest ratings in the first part of the study. Strongly accentuated values that designers tend to take into account while 
designing for the elderly were mapped and categorized in five groups: autonomy, social inclusion, privacy, trust, and dignity.

\subsection{The initial prototype}

A toolkit prototype was proposed for designers to integrate value based approach into the ideation process. Design fiction $[5,6]$ and Bootlegging technique [7] were chosen for the initial design toolkit prototype, based on reviewing a number of design tools and methods. Five design fiction scenarios were written about the context of independent aging supported by technology. The aim of the scenarios was to push readers to think about possible unexpected effects of handing control into smart systems. Design fiction was also expected to encourage critical thinking and to provoke a discussion around the values by presenting possible negative or ambiguous outcomes.

Another component of the proposed toolkit was Bootlegging technique [7] which is a brainstorming technique that provides an opportunity to explore promising ideas as unexpected juxtapositions. It is a fast paced tool for ideating that is helpful for thinking out of the box and come up with unexpected ideas which might be meaningful and might have a design potential.

\subsection{Refining the toolkit in ideation workshops}

The toolkit was iteratively refined in four ideation workshops. The workshops took place over three weeks.

First workshop. The participants were seven design students from two different universities and six different nationalities. First workshop started with participants reading the design fiction scenarios to make them familiar with the context and to hint how the lack of values might be uncomfortable for the users. They were prompted to identify the values in the scenarios and to write them onto post-it notes to use during the following Bootlegging session. The Bootlegging categories were activities, technologies and values. Participants filled the other two categories with relevant content in short brainstorming sessions by writing the technologies and activities on post-it notes. They combined random items from different categories to come up with unexpected juxtapositions and used dot-mocracy (democratic voting within teams) to identify the most promising ideas to develop further. Observing the workshop demonstrated that the values identified from the scenarios were too ambiguous for the participants they had difficulties agreeing on the meanings of the values and some of them seamed to be overlapping. This motivated us to provide value cards with short definitions of the values for the next edition of the workshops.

Second workshop. The participants of the workshop were 7 design professionals from a UX consultancy. The main goal was testing the value card 
set. 18 values were printed out with short descriptions. The structure of the workshop was the same, except that in addition to values identified and discussed from the design fiction scenarios, the participants also received the value cards for Bootlegging. The second workshop demonstrated that at this point we had too many different value cards that were difficult for the participants to categorize and use. Another development in the second workshop was that the participants used the value cards after the ideation process to review their ideas and refine them. This step was included into the structure of the next workshops as value review. The review enabled to extend the design idea and integrate multiple values. In addition, a value map was composed for the next workshop to categorize and explain the value cards and the relations between them.

Third workshop. The participants of the workshops were three design students. The goal of the third workshop was testing the main structure of the workshop that now included value-reviewing as well as the value map. The participants found the review session relevant and helpful for elaborating their design ideas and integrating more values. However, they could not relate to the categories on the provided value map. Thus, to understand how designers perceive the values, a card sorting method was applied online after the workshop. Nine participants form the three conducted workshops participated in the card sorting. The result of card sorting enabled to divide the values in three very broad categories: core values, values related to social needs and nice to have values. For the next edition, the values were categorized accordingly and printed on one value list with values on one side and definitions on the other side of the list.

Fourth workshop. The participants of the last workshop were four design students. The goal of the workshop was testing the value-reviewing session with the categorized value lists. The list was perceived as a useful tool for extending the design ideas and integrating more values to the concept.

\section{Results: a value sensitive toolkit}

The result of the described research through design process is an ideation toolkit for designers, that aims to endorse value-based design of smart habitats for elderly. The work also provides an overview of the tool development process for reflective purposes. The design toolkit comprises of detailed instructions on design fiction, bootlegging and value review and offers a compact and efficient framework to tackle design challenges related to aging in place and smart habitats for the elderly. The design toolkit has four main steps:

1) Design Fiction Scenarios. The first session is composed of a design fiction set which contains 5 design fiction scenarios that focuses on the issues that the elderly face when living in their smart homes. The main goal of this step is activating inspiration and critical thinking. 


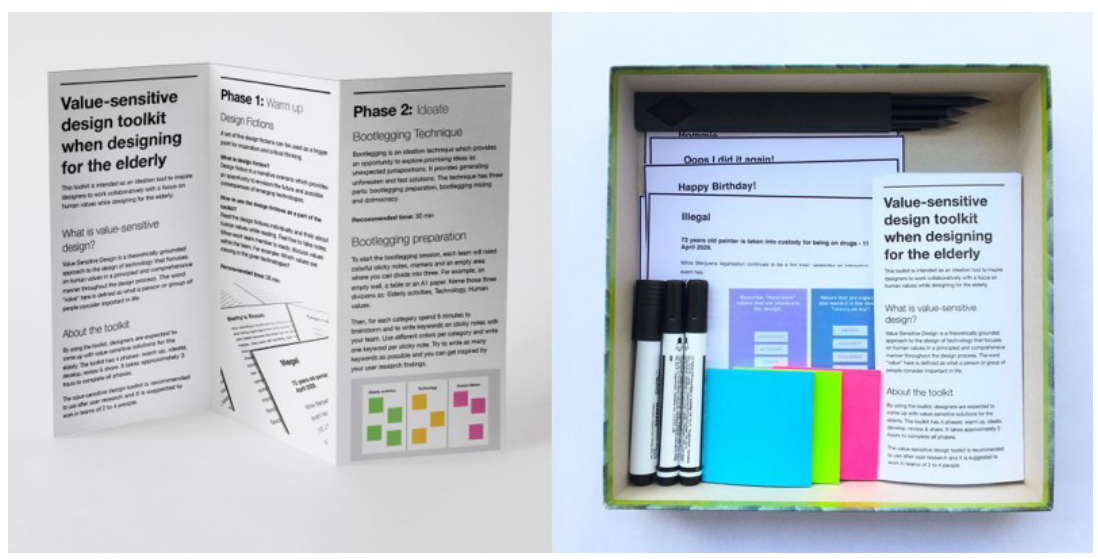

Fig. 1: The design toolkit prototype and instructions.

2) Bootlegging Session. The second step is a brainstorming technique called Bootlegging and it provides an opportunity to explore promising ideas as unexpected juxtapositions. This technique has been modified to bridge ideation with previous user research and to keep focus on the values when ideating design ideas.

3) Developing a design concept. The third step is developing a design concept as a team; the design concept is developed based on one of the bootlegging combinations from the previous step. In this session, the participants are expected to generate ideas and solutions through desired format such as sketching, prototyping, enacting, etc. and create a design concept in order to support aging in place.

4) Value reviewing. As the last step in ideation, a value list is distributed to the teams to review their design concept. The goal of this step is to include more values to their design concept and to refine and extend the idea. The mapped value list is intended to be used as a prompt for discussion and kept nearby also for the later design and development phases in the project.

\section{Discussion and future work}

This process has shown that designers can enhance the design solutions by improving the ideation session within the design process and come up with more value-driven ideas using the proposed design toolkit. The qualitative data gathered from the participants of the study has shown that starting with a critical point of view and practicing Bootlegging technique are forming a promising way to develop creative ideas. The value-review phase provides a value list as a discussion starter to further extend the idea and include a broader range of values. The toolkit development was not related to an actual design project. As future work we would like to keep refining it in the context of an human centered design project where the toolkit could build on conducted user research. 


\section{References}

1. David Siegel and Susan Dray. 2019. The map is not the territory: empathy in design. Interactions 26, 2 (February 2019), 82-85.

2. Osterwalder, A., Pigneur, Y., Bernarda, G., Smith, A. (2014). Value proposition design: How to create products and services customers want. John Wiley, Sons.

3. Zimmerman, J., Forlizzi, J., Evenson, S. (2007, April). Research through design as a method for interaction design research in HCI. In Proceedings of the SIGCHI conference on Human factors in computing systems (pp. 493-502). ACM.

4. Zimmerman, J., Forlizzi, J., Evenson, S. (2007, April). Research through design as a method for interaction design research in HCI. In Proceedings of the SIGCHI conference on Human factors in computing systems (pp. 493-502). ACM.

5. Lindley, J., Coulton, P. (2015, July). Back to the future: 10 years of design fiction. In Proceedings of the 2015 British HCI Conference (pp. 210-211). ACM.

6. Bleecker, J. (2009). Design Fiction: A short essay on design, science, fact and fiction. Near Future Laboratory, 29.

7. Holmquist, L. E. (2008, October). Bootlegging: multidisciplinary brainstorming with cut-ups. In Proceedings of the Tenth Anniversary Conference on Participatory Design 2008 (pp. 158-161). Indiana University.

8. Marek K, Rantz M (2000) Aging in place: a new model for longterm care. Nurs Admin Q 24(3): 111

9. AARP. (2005). Beyond 50.05: A report to the nation on livable communities: Creating environments for successful aging.

10. Birnholtz, J., Jones-Rounds, M. (2010, April). Independence and interaction: understanding seniors' privacy and awareness needs for aging in place. In Proceedings of the SIGCHI Conference on Human Factors in Computing Systems (pp. 143-152). ACM.

11. Altendorf, A and Schreiber, J. Assistive Technology in Dementia Care: Methodological Issues in Research Design. Journal of Assistive Technologies 9, 1 (2015), 3847.

12. Little, L., Briggs, P. (2009, June). Pervasive healthcare: the elderly perspective. In Proceedings of the 2nd International Conference on Pervasive Technologies Related to Assistive Environments (p. 71). ACM.

13. Denning, T., Borning, A., Friedman, B., Gill, B. T., Kohno, T., Maisel, W. H. (2010, April). Patients, pacemakers, and implantable defibrillators: Human values and security for wireless implantable medical devices. In Proceedings of the SIGCHI Conference on Human Factors in Computing Systems (pp. 917-926). ACM.

14. Yi, J. S. Qndreview: Read 100 chi papers in 7 hours. In CHI 14 Extended Abstracts on Human Factors in Computing Systems, CHI EA 14 (New York, NY, USA, 2014), 805814. ACM. 